\title{
Using associated production of top quarks and neutral bosons to probe standard model couplings and search for new physics
}

\author{
Knut Zoch ${ }^{1, *}$ on behalf of the ATLAS Collaboration \\ II. Physikalisches Institut, Georg-August-Universität Göttingen, \\ Friedrich-Hund-Platz 1, 37077 Göttingen, Germany \\ E-mail: kzoch@uni-goettingen.de
}

The unprecedentedly large integrated luminosity accumulated by the ATLAS experiment from proton-proton collisions at a centre-of-mass energy of $\sqrt{s}=13 \mathrm{TeV}$ at the Large Hadron Collider (LHC) allows the study of rare Standard Model (SM) processes. The associated production of top-quark pairs or single top quarks and neutral bosons is such an example: it directly probes topquark couplings to photons and $Z$ bosons and tests for deviations from the SM. Three such recent measurements are presented, all of which exploit the full dataset of the most recent Run 2 of the LHC, corresponding to $139 \mathrm{fb}^{-1}$ of integrated luminosity. The cross-sections for the production of top-quark pairs in association with a photon $(t \bar{t} \gamma)$ or with a $Z$ boson $(t \bar{t} Z)$ are measured both inclusively and differentially as functions of kinematic observables characterising the $t \bar{t}+$ boson system. The measurements are compared to predictions obtained by next-to-leading order (NLO) + parton-shower (PS) Monte Carlo simulations and fixed order NLO calculations, respectively. In a third measurement, the cross-section of single top-quark production in association with a $Z$ boson $(t Z q)$ probes two SM couplings in the same process, $t-Z$ and $W-Z$, and it is a background to the rare associated production of a single top quark and a Higgs boson. A neural network is used to improve the background rejection and to extract the signal. The measured cross-section is compared to the NLO SM prediction.

40th International Conference on High Energy physics - ICHEP2020

July 28 - August 6, 2020

Prague, Czech Republic (virtual meeting)

\footnotetext{
${ }^{1}$ Supported by a doctoral scholarship of the Studienstiftung des Deutschen Volkes.

${ }^{*}$ Speaker
} 


\section{Introduction}

The top quark discovered in 1995 plays a unique role in the Standard Model (SM) of elementary particles due to its large mass of about $173 \mathrm{GeV}$ and the corresponding Yukawa coupling-strength parameter that is close to unity. The production of top quarks in association with neutral vector bosons is of particular interest as these processes give access to the top-boson coupling vertices $(t-V)$. One can write down a generalised, effective Lagrangian for these vertices as

$$
\Gamma_{\mu}^{V}\left(q^{2}\right)=\gamma_{\mu} F_{1}^{V}\left(q^{2}\right)+\frac{i \sigma_{\mu \nu} q^{v}}{2 m_{t}} F_{2}^{V}\left(q^{2}\right)+\gamma_{\mu} \gamma_{5} F_{3}^{V}\left(q^{2}\right)+\frac{i \sigma_{\mu \nu} q^{v}}{2 m_{t}} \gamma_{5} F_{4}^{V}\left(q^{2}\right)
$$

where $F_{1}^{V}, F_{2}^{V}, F_{3}^{V}, F_{4}^{V}$ denote form factors of the different contributions, $m_{t}$ is the mass of the top quark, and $q^{2}$ is the transferred energy-momentum. At leading order (LO) in QCD and electroweak theory in the SM, the coupling vertex between the top quark and the photon is purely vectorial, i.e. $F_{1}^{V} \equiv Q_{t}^{2}$ with the electromagnetic charge $Q_{t}$ of the top quark, and all other form factors vanishing. The coupling to the $Z$ boson receives contributions from the $S U(2)$ weak-isospin field $W_{\mu}^{3}$ and the $U(1)$ hypercharge field $A_{\mu}$, thus resulting in non-zero vectorial and axial-vectorial components $F_{1}^{V}$ and $F_{3}^{V}$. Tensor-like contributions from $F_{2}^{V}$ and $F_{4}^{V}$ are only expected from higher-order loop corrections, but vanish at tree level for both bosons. Many beyond-SM theories predict modifications of the strength or structure of $t-V$ couplings, e.g. added tensor-like contributions, which make studies of these vertices interesting for probing for possible non-SM physics.

Here, three measurements performed with the ATLAS experiment [1] at the Large Hadron Collider are summarised that use $139 \mathrm{fb}^{-1}$ of integrated luminosity, i.e. the full Run 2 dataset: a precision measurement of $t \bar{y} \gamma$ production in the electron-muon final state [2], a precision measurement of $t \bar{t} Z$ production in trilepton and tetralepton final states [3] and the observation of the associated production of a top quark and a $Z$ boson $(t Z q)[4]$.

\section{Top-quark pairs in association with photons}

The production of a top-quark pair, where a photon is radiated by one of the two top quarks, gives access to the $t-\gamma$ coupling. However, the photon may also be radiated by another charged particle of the interaction, e.g. by initial-state quarks or final-state leptons, which yields an identical final state. The ATLAS Collaboration first measured $t \bar{t} \gamma$ production at $\sqrt{s}=13 \mathrm{TeV}$ with Run 2 data taken in 2015 and 2016, corresponding to $36 \mathrm{fb}^{-1}$ of integrated luminosity, where both $\ell+$ jets and dilepton channels were probed [5]. Now, the ATLAS Collaboration has conducted a precision measurement of $t \bar{t} \gamma$ in the $e \mu$ channel using the full Run 2 dataset where fiducial inclusive and differential cross-sections in several observables are compared to a fixed-order theory prediction computed at next-to-leading order (NLO) in QCD [6, 7]. For consistency with the theory prediction that includes all off-shell contributions and interference effects, i.e. also singly-resonant and nonresonant diagrams, a combined $t \bar{t} \gamma+t W \gamma$ measurement was performed.

Events are selected by requiring one electron and one muon of opposite electric charge. Two or more anti- $k_{t}$ jets with a radius parameter of $R=0.4$ are required, of which at least one must be tagged as a $b$-jet with the MV2c10 algorithm at an $85 \%$ fixed-efficiency operating point. In addition, the event must contain one photon with $p_{\mathrm{T}}>20 \mathrm{GeV}$. Using a binned profile 

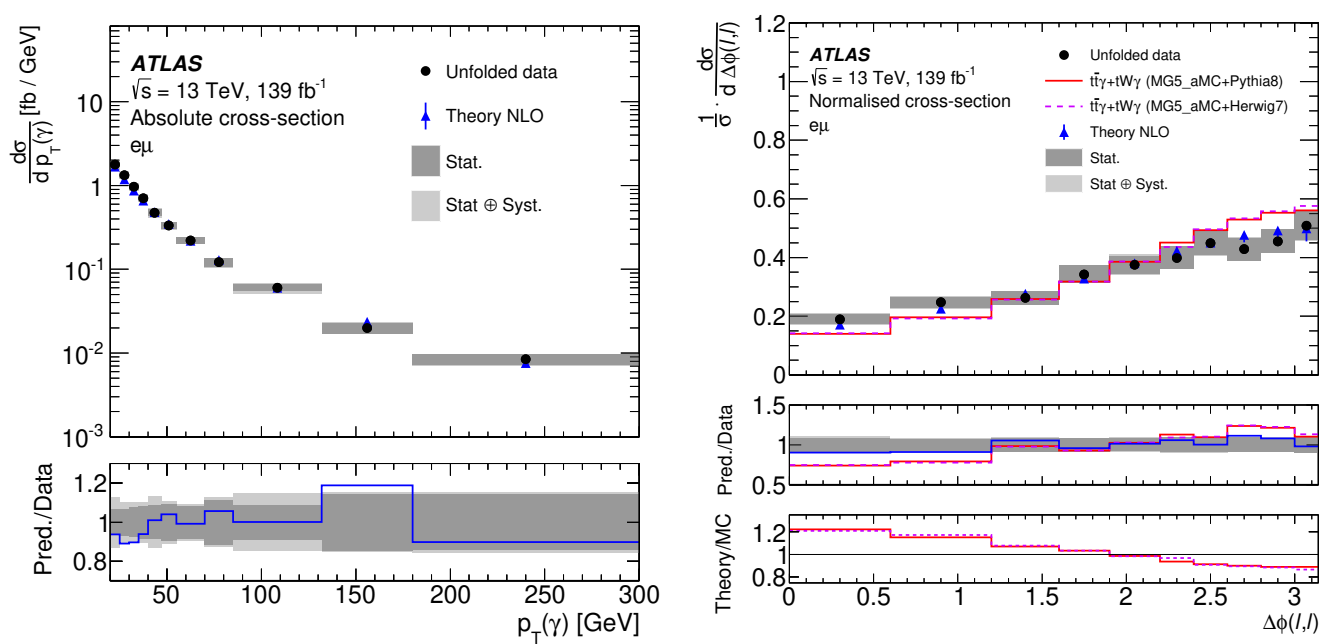

Figure 1: Examples of differential cross-sections of $t \bar{t} \gamma$ production in the $e \mu$ final state [2]: on the left as a function of the photon transverse momentum (absolute cross-section), on the right as a function of the difference in the azimuthal angles of the two leptons (cross-section normalised to unity).

likelihood fit to the distribution of the observable $S_{\mathrm{T}}$, the scalar sum of the transverse momenta of all reconstructed objects in the event including $E_{\mathrm{T}}^{\text {miss }}$, the measured fiducial cross-section amounts to $\sigma^{\text {fid }}(t \bar{t} \gamma \rightarrow e \mu)=39.6 \pm 0.8$ (stat) ${ }_{-2.2}^{+2.6}$ (syst) fb. This is to be compared against the fixed-order theory prediction $[6,7]$ of $\sigma_{\mathrm{NLO}}^{\mathrm{fid}}=38.50_{-2.18}^{+0.56}(\mathrm{scale})_{-1.18}^{+1.04}(\mathrm{PDF}) \mathrm{fb}$. Dominant uncertainties of the fiducial inclusive measurement are the modelling uncertainties of signal and background processes and the ATLAS luminosity measurement.

The ATLAS data was also unfolded to parton level using an iterative approach based on Bayes' theorem [8] and compared against the fixed-order NLO computation and LO+PS Monte Carlo simulations differentially. Examples of two unfolded spectra are shown in Figure 1. The NLO theory computation shows good agreement with ATLAS data in all unfolded observables, whereas the LO+PS Monte Carlo simulation has difficulties to describe some of the observables accurately.

\section{Top-quark pairs in association with $Z$ bosons}

After a first measurement of the $t \bar{t} Z$ cross-section at $\sqrt{s}=13 \mathrm{TeV}$ with $36 \mathrm{fb}^{-1}$ [9], ATLAS has now performed a measurement of the inclusive and differential cross-sections in the trilepton and tetralepton channels using the full Run 2 dataset [3]. Two non-overlapping trilepton regions with different $b$-tag requirements are defined for the inclusive measurement to maximise the precision of the measurement and to suppress $W Z$ background contributions. For the differential measurement, one common trilepton region is defined to exploit the maximum statistics. The tetralepton channel uses four signal regions that differ by the number of $b$-tagged jets (exactly one vs. two or more at $85 \%$ efficiency) and lepton-flavour requirements (two electrons and two muons vs. four leptons of the same flavour). Additional control regions for the $Z Z$ (with four leptons) and $W Z$ (with three leptons) backgrounds are defined.

A simultaneous profile likelihood fit in all trilepton and tetralepton signal regions as well as in the background control regions is performed. The obtained inclusive $t \bar{t} Z$ cross-section amounts 

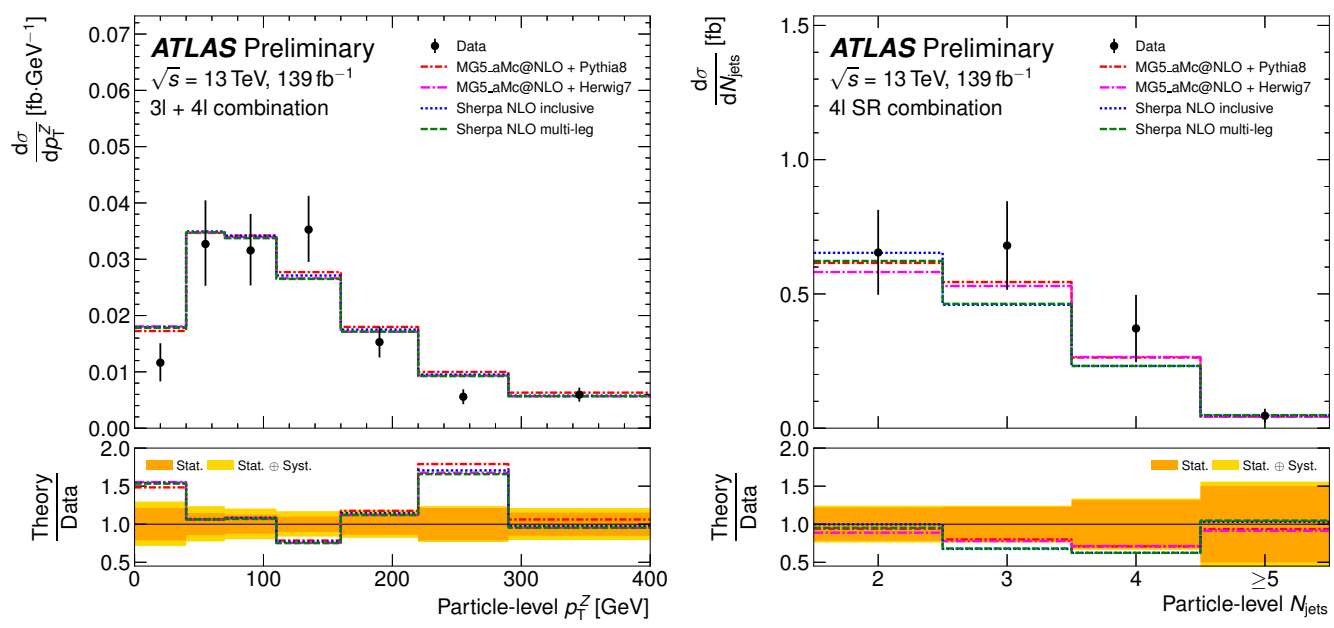

Figure 2: Examples of differential cross-sections of $t \bar{t} Z$ production in trilepton and tetralepton final states [3]: on the left as a function of the transverse momentum of the reconstructed $Z$ boson at particle level, on the right as a function of the jet multiplicity at particle level.

to $\sigma(t \bar{t} Z)=1.05 \pm 0.05$ (stat) \pm 0.09 (syst) pb, which is in good agreement with the NLO + nextto-leading-log prediction [10] of $\sigma^{\mathrm{NLO}+\mathrm{NNLL}}(t \bar{t} Z)=0.863_{-0.09}^{+0.07}$ (scale $) \pm 0.03\left(\mathrm{PDF}+\alpha_{S}\right) \mathrm{pb}$. The fit results are also obtained individually in the trilepton and tetralepton channels and compared against the combined-channel result for consistency. Dominant uncertainties include the modelling of signal and background processes, e.g. of the $t \bar{t} Z$ parton shower.

The same iterative approach as in the $t \bar{t} \gamma$ measurement is used to unfold ATLAS data to parton and particle level [8]. Differential cross-sections of several observables are obtained, out of which only two examples are shown in Figure 2: the transverse momentum of the reconstructed $Z$ boson in the combined trilepton and tetralepton channels at particle level, an observable that is sensitive to beyond-SM contributions in the $t-Z$ coupling, and the jet multiplicity in the tetralepton channels at particle level, which gives valuable insights into the modelling of high jet multiplicities in Monte Carlo simulation. Most of the observables are found to show good agreement between data and the predictions. However, among others, the distribution of the transverse momentum of the reconstructed $Z$ boson shows notable disagreement between data and the prediction, resulting in low $p$-values in the performed compatibility tests.

\section{Single top quarks in association with $Z$ bosons}

The production of a single top quark in association with a $Z$ boson $(t Z q)$ is possible through multiple tree-level diagrams that contain the $t-Z$ and the $Z-W$ coupling, as shown in the Feynman diagrams in Figure 3. First evidence of this process had been seen by ATLAS at $\sqrt{s}=13 \mathrm{TeV}$ using Run 2 data corresponding to $36 \mathrm{fb}^{-1}$ of integrated luminosity [11]. The process was observed by the CMS Collaboration using Run 2 data from 2016 and 2017 [12]. Now, ATLAS has reported an observation of $t Z q$ production in the trilepton channels using the full Run 2 dataset [4].

A pre-selection is applied to all regions as follows: three charged leptons with at least one opposite-charge, same-flavour lepton pair with $\left|m_{\ell \ell}-m_{Z}\right|<10 \mathrm{GeV}$ are required. For $\mu \mu e$ and 

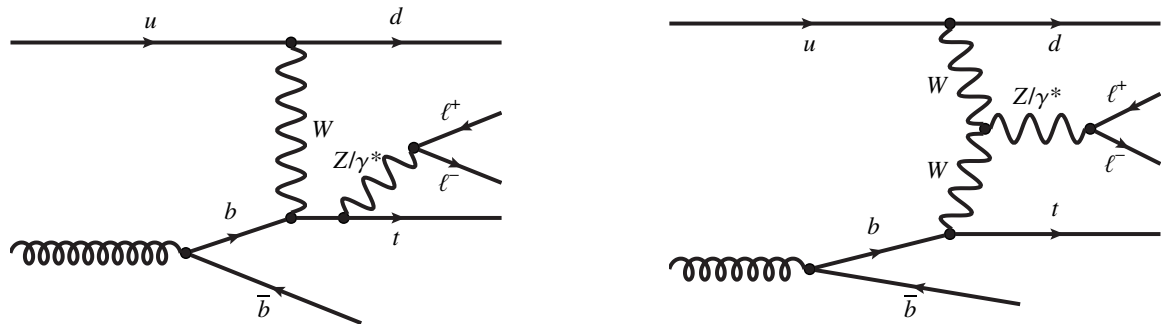

Figure 3: Representative tree-level Feynman diagrams for the production of a single top quark in association with a $Z$ boson involving the $t-Z$ and the $Z-W$ coupling.

$e e \mu$ final states, this is unambiguous, whereas for the $\mu \mu \mu$ and $e e e$ final states the two leptons with an invariant mass closest to the $Z$ boson mass are paired. Non-resonant lepton pairs with $m_{\ell \ell}>30 \mathrm{GeV}$ are included in the used simulation. Two signal regions are defined by requiring one $b$-tagged jet plus one untagged jet, and two $b$-tagged jets plus one untagged jet, respectively. The $70 \%$ fixed-efficiency operating point of the MV2c10 algorithm is used. In addition, the untagged jet is expected to be emitted at high absolute pseudorapidity. Control regions for $t \bar{t} Z$ events are defined by increasing the jet and $b$-jet multiplicity requirements and for diboson events by applying a veto on $b$-tagged jets. Two $t \bar{t}$ control regions are derived from the signal regions by selecting an oppositecharge different-flavour lepton pair instead of a same-flavour lepton pair. The normalisation of the background processes floats freely in the fit and is determined through the control regions. The shape of the fake-lepton estimate (e.g. $t \bar{t}$ and $Z+$ jets) is derived purely from Monte Carlo simulation, however, statistics of this estimate are enriched from a dilepton event selection with two $b$-tagged jets, where one of the $b$-jets is replaced by a lepton as a proxy. The energies and polar angles are replaced according to Monte Carlo studies.

Neural networks to maximise the signal-background separation are trained separately for the two signal regions using 15 input variables each. The best separating input variables include the invariant mass of the $b$-jet and the untagged jet as well as the reconstructed top-quark mass. A simultaneous profile likelihood fit of the neural-network outputs in the signal regions and other observables in the control regions is performed to extract a $t Z q$ cross-section of $\sigma(t Z q)=97 \pm$ 13 (stat) \pm 7 (syst) fb. This is compatible with the NLO theory prediction of $\sigma^{\mathrm{NLO}}(t Z q)=102_{-2}^{+5} \mathrm{fb}$. Both, the expected and measured significances considering a background-only hypothesis are well above five standard deviations. Apart from data statistics being the limiting factor, the dominant uncertainties include the modelling and the rate of the prompt-lepton background.

\section{Summary}

The large dataset of $139 \mathrm{fb}^{-1}$ of integrated luminosity, collected with the ATLAS experiment during Run 2 of the Large Hadron Collider, has enabled precision measurements of final states with top quarks and neutral bosons. ${ }^{1}$ Three recent measurements using this dataset were summarised here: a $t \bar{t} \gamma$ measurement in the $e \mu$ final state [2], a $t \bar{t} Z$ measurement in the trilepton and tetralepton final states [3] and the observation of $t Z q$ production [4]. Good agreement with the SM predictions is found in the inclusive measurements and in differential spectra. State-of-the-art fixed-order

\footnotetext{
${ }^{1}$ The full list of top-quark results is available at: https://twiki.cern.ch/twiki/bin/view/AtlasPublic/TopPublicResults
} 
computations and NLO+PS Monte Carlo simulations provide good descriptions of the data spectra, whereas LO+PS Monte Carlo simulations describe some of the observables insufficiently. In summary, these ATLAS measurements have put the SM predictions under stringent test, but reducing the uncertainties associated to the modelling of signal and background will be pivotal for increasing the sensitivity to possible anomalies in the $t-V$ couplings.

\section{References}

[1] ATLAS Collaboration, The ATLAS Experiment at the CERN Large Hadron Collider, JINST 3 (2008) S08003

[2] ATLAS Collaboration, Measurements of inclusive and differential cross-sections of combined $t \bar{t} \gamma$ and $t W \gamma$ production in the e $\mu$ channel at $13 \mathrm{TeV}$ with the ATLAS detector, JHEP 09 (2020) 049 [arXiv:2007.06946 [hep-ex]]

[3] ATLAS Collaboration, Measurements of the inclusive and differential production cross sections of a top-quark-antiquark pair in association with a $\mathrm{Z}$ boson at $\sqrt{s}=13 \mathrm{TeV}$ with the ATLAS detector, ATLAS-CONF-2020-028 (2020), http://cds.cern.ch/record/2725734

[4] ATLAS Collaboration, Observation of the associated production of a top quark and a $Z$ boson in pp collisions at $\sqrt{s}=13 \mathrm{TeV}$ with the ATLAS detector, JHEP 07 (2020) 124 [arXiv:2002.07546 [hep-ex]]

[5] ATLAS Collaboration, Measurements of inclusive and differential fiducial cross-sections of $t \bar{t} \gamma$ production in leptonic final states at $\sqrt{s}=13 \mathrm{TeV}$ in ATLAS, Eur. Phys. J. C 79 (2019) 382 [arXiv:1812.01697 [hep-ex]]

[6] G. Bevilacqua et al., Hard Photons in Hadroproduction of Top Quarks with Realistic Final States, JHEP 10 (2018) 158 [arXiv:1803.09916 [hep-ph]]

[7] G. Bevilacqua et al., Precise predictions for $t \bar{t} \gamma / t \bar{t}$ cross section ratios at the LHC, JHEP 01 (2019) 188 [arXiv:1809.08562 [hep-ph]]

[8] G. D'Agostini, A multidimensional unfolding method based on Bayes' theorem, Nucl. Instrum. Meth. A 362 (1995) 487

[9] ATLAS Collaboration, Measurement of the $t \bar{t} Z$ and $t \bar{t} W$ cross sections in proton-proton collisions at $\sqrt{s}=13 \mathrm{TeV}$ with the ATLAS detector, Phys. Rev. D 99 (2019) 072009 [arXiv:1901.03584 [hep-ex]]

[10] A. Kulesza et al., Associated production of a top quark pair with a heavy electroweak gauge boson at NLO+NNLL accuracy, Eur. Phys. J. C 79 (2019) 249 [arXiv:1812.08622 [hep-ph]]

[11] ATLAS Collaboration, Measurement of the production cross-section of a single top quark in association with a $\mathrm{Z}$ boson in proton-proton collisions at $13 \mathrm{TeV}$ with the ATLAS detector, Phys. Lett. B 780 (2018) 557 [arXiv:1710.03659 [hep-ex]] 
[12] CMS Collaboration, Observation of Single Top Quark Production in Association with a $Z$ Boson in Proton-Proton Collisions at $\sqrt{s}=13 \mathrm{TeV}$, Phys. Rev. Lett. 122 (2019) 132003 [arXiv:1812.05900 [hep-ex]] 